\title{
Caracterização imunofenotípica das células plasmáticas em pacientes portadores de mieloma múltiplo
}

Primeira submissão em 28/02/10 Última submissão em 24/05/10 Aceito para publicação em 25/05/10 Publicado em 20/08/10

\section{Immunophenotypic characterization of plasma cells in patients with multiple myeloma}

Luiz Arthur Calheiros Leite'; Manuela S. Almeida²; Elisa S. Kimura³; Jandey G. Bigonha4; Gisele W. B. Colleoni ${ }^{5}$; Maria de Lourdes L. F. Chauffaille; ${ }^{6}$ Mihoko Yamamoto ${ }^{7}$

\begin{tabular}{|c|c|}
\hline & resumo \\
\hline $\begin{array}{l}\text { Plasmócitos } \\
\text { Mieloma múltiplo } \\
\text { Fenótipos aberrantes } \\
\text { Imunofenótipo } \\
\text { Citometria de fluxo } \\
\text { Prognóstico }\end{array}$ & 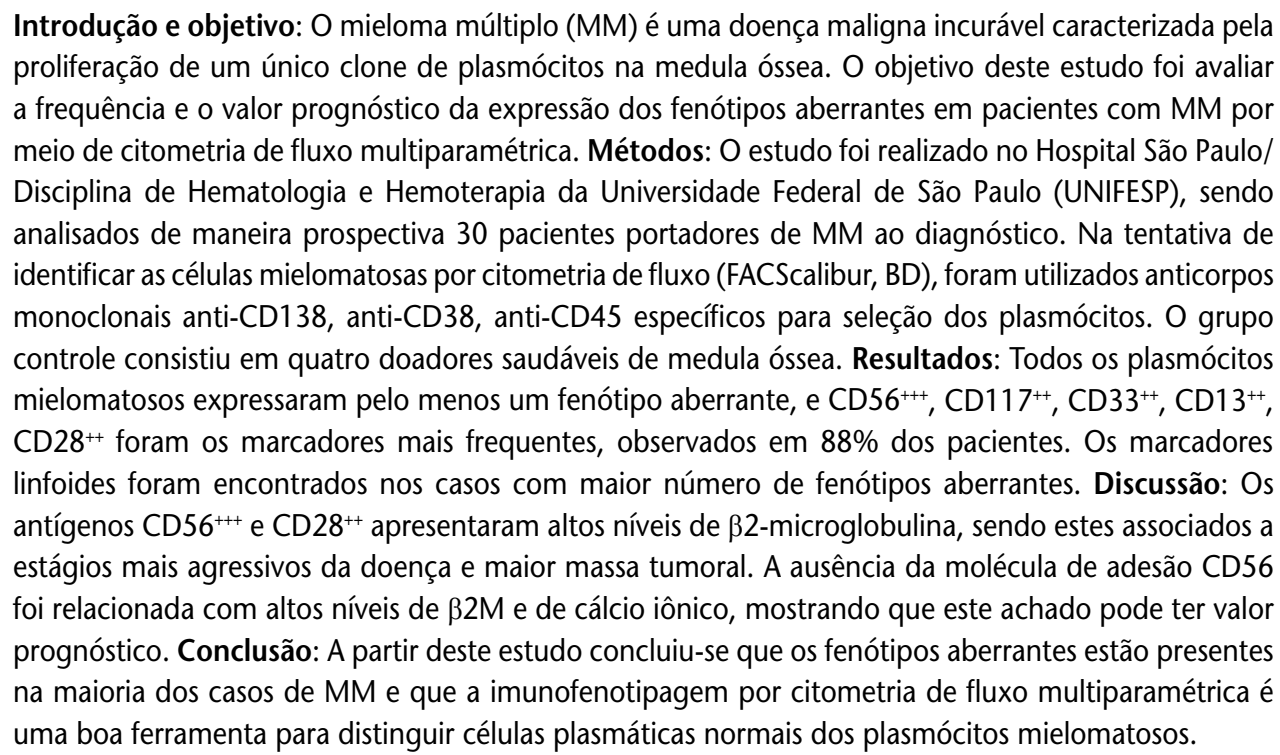 \\
\hline
\end{tabular}

\section{abstract}

Introduction and objective: Multiple myeloma is an incurable malignancy characterized by the proliferation of a single clone of plasma cells in bone marrow. The aim of this study was to evaluate the frequency and prognostic value of the expression of aberrant phenotypes in patients with multiple myeloma by multiparametric flow cytometry. Methods: The study was carried out at Department of Hematology and Hemotherapy of Federal University of São Paulo and 30 patients with MM were analyzed prospectively. In an attempt to identify myeloma cells by flow cytometry (FACSCalibur, BD), specific monoclonal antibodies anti-CD138, anti-CD38 and anti-CD45 were used for the selection of plasma cells. The control group comprised four healthy bone marrow donors. Results: All myeloma plasma cells expressed at least one aberrant phenotype and CD56 $6^{+++}$, $C D 117^{++}, C D 33^{++}, C D 13^{++}$and $C D 28^{++}$markers were more frequently observed in $88 \%$ of patients. Lymphoid markers were found in cases with a higher number of aberrant phenotypes. Discussion: $C D 56^{+++}$and CD28 $8^{++}$ antigens showed high levels of $\beta 2$-microglobulin, which are associated with more aggressive stages of the disease and larger tumor mass. The absence of adhesion molecule CD56 was associated with high levels of $\beta 2 \mathrm{M}$ and calcium ion, showing that this finding may have prognostic value. Conclusion: From this study it was concluded that the aberrant phenotypes are present in most cases of $M M$, and immunophenotyping by multiparametric flow cytometry is a useful tool to distinguish normal plasma cells from myeloma plasma cells. key words

Plasma cells

Multiple myeloma

Aberrant phenotype

Immunophenotype

Flow cytometry

Prognosis

\footnotetext{
1. Mestre em Hematologia pela Escola Paulista de Medicina da Universidade Federal de São Paulo (EPM/UNIFESP); doutorando em Bioquímica pela Universidade Federal de Pernambuco (UFPE); professor de Hematologia da Faculdade de Ciências Biológicas e da Saúde do Centro de Estudos Superiores de Maceió (FCBS/CESMAC).

2. Doutora em Hematologia pela UNIFESP; médica.

3. Especialista em Farmacêutica Bioquímica pela UNIFESP.

4. Mestra em Hematologia pela UNIFESP; médica.

5. Livre-docente pela UNIFESP.

6. Live-docente na UNIFESP.

7. Professora doutora associada da UNIFESP.

Suporte financeiro: Projeto financiado pela Fundação de Amparo à Pesquisa do Estado de São Paulo (FAPESP) sob o Protocolo ño 01/13086-3.
} 


\section{Introdução}

O mieloma múltiplo (MM) é uma neoplasia incurável caracterizada por proliferação de plasmócitos clonais na medula óssea (MO) que pode cursar com alterações imunofenotípicas heterogêneas, além de múltiplas disfunções orgânicas, como produção de grande quantidade de imunoglobulinas, falência renal, hipercalcemia com destruição esquelética, anemia e infecções recorrentes ${ }^{(2,8)}$.

Até algum tempo atrás, a imunofenotipagem no MM era utilizada apenas para fins de pesquisas científicas, porém, com o surgimento de marcadores mais específicos que caracterizam os plasmócitos mielomatosos (CD138 $8^{+++}$, $\mathrm{CD} 38^{++}, \mathrm{CD} 45^{+}$ou CD45, CD56 ${ }^{+++}, \mathrm{CD} 19^{-}$e a restrição de cadeia leve $\kappa$ ou $\lambda$ ), tornou-se possível detectar a expressão dos antígenos de superfície e citoplasmáticos nos plasmócitos de pacientes com MM e diferenciá-los claramente dos plasmócitos normais policlonais ${ }^{(1,16)}$.

A população de células plasmáticas normais é caracterizada pela expressão de marcadores de superfície comuns em linfócitos B, como CD19, CD20, CD22 e CD45, e pela ausência do CD56. Já as células mielomatosas comumente não expressam o CD19 e apresentam expressão fraca ou ausente do CD45, além de a maioria dos casos expressar fortemente o antígeno CD56(6).

Estudos relatam que outros marcadores de superfície estão presentes nos plasmócitos mielomatosos, como os antígenos mieloides (CD117, CD33, CD14, CD13). Além disso, os antígenos associados à linhagem linfoide (CD10, CD20, CD22, CD28) são encontrados em baixa frequência nos plasmócitos clonais. Estes antígenos geralmente estão ausentes em plasmócitos normais, sendo denominados fenótipos de expressão aberrante quando expressos em células mielomatosas ${ }^{(9,12)}$.

Este estudo tem por objetivo determinar a frequência e o valor prognóstico dos fenótipos aberrantes em pacientes portadores de MM, utilizando a imunofenotipagem por citometria de fluxo multiparamétrica.

\section{Casuística e método}

A presente pesquisa foi desenvolvida na disciplina de Hematologia e Hemoterapia da Escola Paulista de Medicina da Universidade Federal de São Paulo (EPM/UNIFESP) com colaboração do Hospital do Servidor Público Estadual de São Paulo Francisco Morato de Oliveira (HSPE-FMO), no período de março de 2003 a dezembro de 2006.
Para isso foram analisadas 30 amostras de $\mathrm{MO}$ de pacientes portadores de MM ao diagnóstico (sem tratamento quimioterápico prévio e/ou transplante de medula óssea). A idade média foi 62,5 anos, variando entre 27 e 79 anos, e o sexo masculino prevaleceu numa proporção 2,5:1. Foram utilizados como controle quatro pacientes saudáveis doadores de $\mathrm{MO}$. $\mathrm{O}$ estudo foi previamente encaminhado ao Comitê de Ética e Pesquisa da EPM/UNIFESP, tendo sido aprovado (Protocolo oㅜ 1407/04).

De acordo com o sistema de estadiamento de Durie\&Salmon, descritos na Tabela 1, $90 \%$ dos pacientes pertenciam ao estágio III, $7 \%$ ao estágio II e apenas 3\% foram classificados como estágio I. Além disso, foi utilizado o sistema internacional de estadiamento do mieloma múltiplo, descrito na Tabela 2, em que o valor médio da $\beta 2$-microglobulina ( $\beta 2 \mathrm{M}$ ) foi de $7,6 \mathrm{mg} / \mathrm{dl}$ e a média da albumina sérica, inferior a 3,5 g/dl em $70 \%$ dos casos. Segundo o sistema internacional, apenas 3\% dos pacientes estudados pertenciam ao estágio I, 33\% ao estágio II e 64\% ao estágio III.

Para determinação dos isótipos de imunoglobulinas foram utilizadas técnicas de imunofixação, na qual $60 \%$ dos casos estudados pertenciam ao isótipo de cadeia pesada IgG e $70 \%$ das cadeias leves secretadas eram kappa. Foi também determinado outro parâmetro associado a lesões osteolíticas: o cálcio iônico, que teve como média o valor de $1,4 \mathrm{mg} / \mathrm{dl}$, variando entre 1,02 e 2,33 mg/dl.

\section{Estratégias para o estudo imunofenotípico dos plasmócitos}

Para a imunofenotipagem as amostras de medula óssea foram coletadas em tubos contendo o anticoagulante ácido etilenodiaminotetracético (EDTA). Posteriormente, as amostras de $\mathrm{MO}$ foram incubadas com os anticorpos monoclonais por 15 minutos em temperatura ambiente e no escuro. Para seleção dos plasmócitos e estudos dos fenótipos aberrantes foi testado grande número de anticorpos associados a quatro fluorocromos: CD138 isotiocianato de flureceína (FITC), CD28/FITC, CD10/FITC, CD14/FITC, CD57/FITC, CD34/FITC,

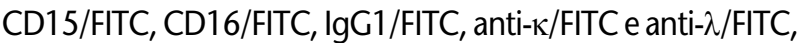
IgG2/ficoeritrina (PE), CD138/PE, CD56/PE, CD20/PE, CD19/ $\mathrm{PE}, \mathrm{CD} 22 / \mathrm{PE}, \mathrm{CD} 117 / \mathrm{PE}, \mathrm{CD} 13 / \mathrm{PE}, \mathrm{CD} 33 / \mathrm{PE}, \mathrm{CD} 40 \mathrm{PE}, \mathrm{CD} 45 /$ proteína clorofilada peridinina (PerCP), CD38/alloficocianina (APC), CD19/APC. Todos os anticorpos foram adquiridos pela empresa Becton Dickinson/BD, San José, CA, EUA.

Após a incubação, os eritrócitos da amostra de $\mathrm{MO}$ foram lisados com um tampão hemolítico (FACS lysing 


\section{Tabela 1 Estadiamento do MM de acordo com os critérios de Durie\&Salmon}

\begin{tabular}{l} 
Estágio I (pequena massa tumoral, $<0,6 \times 10^{12}$ células $/ \mathrm{m}^{2}$ ) \\
Hemoglobina $>10 \mathrm{~g} / \mathrm{dl}$ \\
IgG $<5 \mathrm{~g} / \mathrm{dl}$; IgA $<3 \mathrm{~g} / \mathrm{dl}$; proteinúria de BJ na urina $<4 \mathrm{~g} / 24 \mathrm{~h}$ \\
Cálcio sérico normal \\
Lesões líticas generalizadas \\
Estágio II (massa tumoral intermediária) \\
Achados entre estágios I e III \\
Estágio III (massa tumoral, > 1,2 x $10^{12}$ células $/ \mathrm{m}^{2}$ ) \\
Hemoglobina $<8,5 \mathrm{~g} / \mathrm{dl}$ \\
IgG $>7 \mathrm{~g} / \mathrm{dl} ;$ IgA $>5 \mathrm{~g} / \mathrm{dl}$; proteinúria de BJ na urina $>12 \mathrm{~g} / 24 \mathrm{~h}$ \\
Cálcio > $12 \mathrm{mg} / \mathrm{dl}$ \\
Lesões líticas generalizadas \\
Critérios acrescidos \\
A - Creatinina $<2 \mathrm{mg} / \mathrm{dl}$ \\
B - Creatinina $>2 \mathrm{mg} / \mathrm{dl}$ \\
\hline MM: mieloma múltiplo; IgG: imunoglobulina de classe G; IgA: imunoglobulina de classe A; BJ: Bence Jones.
\end{tabular}

Tabela 2 Sistema internacional de estadiamento do MM

\begin{tabular}{ccc}
\hline Estágios & Critérios & Sobrevida média (meses) \\
\hline I & $\beta_{2}$ microglobulina $<3,5 \mathrm{mg} / \mathrm{l}$ & 62 \\
Albumina sérica $>3,5 \mathrm{~g} / \mathrm{dl}$ & \\
$\mathrm{II}$ & $\beta_{2}$ microglobulina $3,5-5,5 \mathrm{mg} / \mathrm{l} \mathrm{ou}$ \\
& $\beta_{2}$ microglobulina $<3,5 \mathrm{mg} / \mathrm{l} \mathrm{com}$ \\
albumina sérica $<3,5 \mathrm{~g} / \mathrm{dl}$ & 44 \\
$\mathrm{III}$ & $\beta_{2}$ microglobulina $>5,5 \mathrm{mg} / \mathrm{l}$ & \\
\hline
\end{tabular}

MM: mieloma múltiplo.

solution, BD) e lavados com solução de tampão fosfato (PBS) para remover o excesso de imunoglobulinas séricas. Para que as amostras pudessem ser adquiridas foi necessário que ocorresse fixação dos anticorpos por meio do uso de $500 \mu \mathrm{l}$ de uma solução de metanol/fomaldeído a 1\%. Quando o exame microscópico da $\mathrm{MO}$ revelava poucos plasmócitos, utilizava-se uma solução de Ficoll-Hypaque, que permitia melhor separação e análise das células mononucleares.

As aquisições e análises imunofenotípicas foram realizadas no citômetro de fluxo FACScalibur (Becton Dickinson, BD) por meio dos programas Cell-Quest e Paint-a-gate. Os plasmócitos mielomatosos foram identificados utilizandose os anticorpos monoclonais CD138 ${ }^{+++}, \mathrm{CD} 38^{++}, \mathrm{CD} 45^{+}$ ou CD45. Os marcadores foram considerados positivos quando eram detectados $20 \%$ ou mais de positividade dos plasmócitos clonais.
Para análise estatística foram utilizados os testes de Fisher, Mann-Whitney e t-Student para determinar a significância estatística e as associações entre os fenótipos aberrantes e os parâmetros laboratoriais. $\mathrm{O}$ valor foi considerado significante quando o $p$ era inferior a 0,05 .

\section{Resultados}

\section{Estudo imunofenotípico dos plasmócitos normais}

Toda a população de células plasmáticas normais expressava os marcadores $\mathrm{CD} 138^{+++}, \mathrm{CD} 38^{++}, \mathrm{CD} 45^{+}, \mathrm{CD} 19^{++}$, $\mathrm{CD}^{2} 0^{++}$e CD22 ${ }^{++}$, era negativa para o CD56 e exibia reatividade tanto para a imunoglobulina de cadeia leve kappa (к) como para lambda ( $\lambda$ ). O valor médio de positividade 
dos plasmócitos para os marcadores CD19 e CD45 foi de $54 \%$ e $50 \%$, respectivamente. Todas as células normais estudadas expressavam o antígeno $\mathrm{CD} 40^{+++}$, enquanto os marcadores CD10, CD13, CD14, CD28, CD33, CD34, CD56, CD57, CD117 e HLA-DR foram negativos em todos os controles normais.

\section{Estudo imunofenotípico dos plasmócitos mielomatosos}

Os marcadores CD138 e CD38 foram positivos em todos os casos de MM e utilizados para estudo dos plasmócitos mielomatosos. Todas as amostras analisadas apresentavam monoclonalidade, sendo $73 \%$ dos casos $\kappa$ e $27 \% \lambda$. Diferente dos indivíduos normais, o marcador CD45 foi identificado em apenas 19\% dos pacientes, com valor médio de células positivas de 54\%, e o CD19 foi positivo em apenas 3\% dos casos, com forte expressão antigênica (86\% das células mielomatosas). O antígeno CD56 apresentava forte expressão em $76,7 \%$ dos casos, com média de expressão de $69 \%$ das células positivas. Outros fenótipos aberrantes também foram frequentemente encontrados nos plasmócitos mielomatosos, como CD117 (60\% dos casos de MM), CD33 (29\%), CD13 (28\%) e CD28 (20\%). Um fato curioso foi que os marcadores associados à linhagem linfoide (CD10, CD19, CD20 e CD22) apresentaram baixa frequência na população estudada, conforme mostra a Tabela 3. Para melhor visualização, a Figura ilustra alguns fenótipos aberrantes presentes nos pacientes portadores de mieloma múltiplo.

\section{Tabela 3 Frequência dos fenótipos aberrantes nos 30 pacientes avaliados}

\begin{tabular}{ccccc}
\hline AcM0 & $n$ & Positividade $n(\%)$ & Média* & Variação \\
CD56 & 30 & $23(76,7)$ & 96,0 & $55-100$ \\
CD117 & 30 & $18(60)$ & 69,9 & $28,7-99,5$ \\
CD33 & 30 & $9(30)$ & 59,7 & $21,1-99,5$ \\
CD13 & 30 & $8(26,7)$ & 44,3 & $20,7-96,9$ \\
CD28 & 30 & $6(20)$ & 57,2 & $20,4-95,9$ \\
CD10 & 30 & $3(10)$ & 30,6 & $28,6-34,2$ \\
CD22 & 30 & $3(8,1)$ & 48 & $33,5-67,8$ \\
CD20 & 30 & $3(10)$ & 65,5 & $29,8-97,9$ \\
CD14 & 25 & $3(12)$ & 40,8 & $28,3-48,2$ \\
HLA-DR & 25 & $1(4)$ & 65,5 & 65,5 \\
\hline
\end{tabular}

Os resultados foram expressos em porcentagem, considerando-se positivos quando o valor excedia $20 \%$.

* Média: valor médio das células mielomatosas positivas; variação: valores mínimo e máximo de células positivas; AcMo: anticorpos monoclonais.
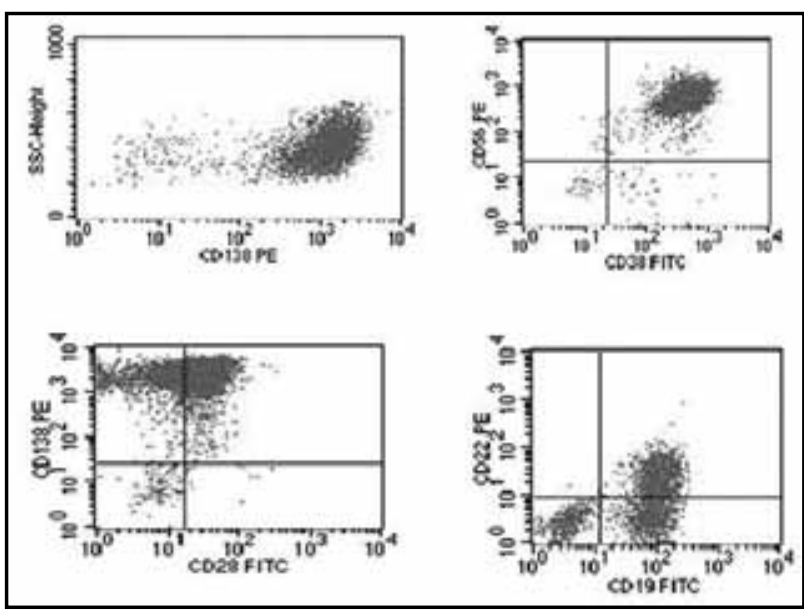

Figura 1 - Expressão aberrante dos marcadores CD56+++, CD28++, CD22++e CD19++ em plasmócitos do MM ao diagnóstico.

*Os plasmócitos foram identificados por meio da forte expressão do $\mathrm{CD}_{138^{++}} \mathrm{e}$ da complexidade celular (SSC, side scatter).

\section{Associação entre os achados clinicolaboratoriais e os fenótipos aberrantes nos pacientes com MM}

De acordo com a Tabela 4, quando se relacionaram os fenótipos aberrantes aos marcadores clinicolaboratoriais, foi observado que $23,3 \%$ dos casos foram negativos para o CD56 e apresentavam altos níveis séricos de $\beta 2 \mathrm{M}$ quando em comparação com os casos $\operatorname{CD}^{2} 6^{+}(p=0,003)$.

Em relação aos casos que não expressavam CD117, observou-se que apresentavam níveis séricos de cálcio ionizável maiores que os pacientes $\mathrm{CD}_{117^{+}}(p=0,02)$. A presença do antígeno CD28 ${ }^{+}$foi observada em $20 \%$ dos pacientes com MM e associada a altos níveis de $\beta 2 \mathrm{M}$ quando em comparação com os pacientes CD28. $(p=0,04)$. 


\section{Relação entre as características clínicas e biológicas dos 30 pacientes com MM com os marcadores}

Tabela 4 CD56, CD28 e CD117

\begin{tabular}{|c|c|c|c|c|c|c|c|c|c|}
\hline Parâmetros & $\begin{array}{l}\text { CD56 } \\
(n=7)\end{array}$ & $\begin{array}{l}\text { CD56 }^{+} \\
(n=23)\end{array}$ & $p$ & $\begin{array}{l}\text { CD28 } \\
(n=24)\end{array}$ & $\begin{array}{l}C D 28^{+} \\
(n=6)\end{array}$ & $p$ & $\begin{array}{l}\text { CD117 } \\
(n=12)\end{array}$ & $\begin{array}{l}\mathrm{CD} 117^{+} \\
(n=18)\end{array}$ & $p$ \\
\hline Idade* & $\begin{array}{c}59,1 \pm \\
3,2\end{array}$ & $\begin{array}{c}63,7 \pm \\
13,5\end{array}$ & & $\begin{array}{c}63,0 \pm \\
13,1\end{array}$ & $\begin{array}{c}61 \pm \\
6,4\end{array}$ & & $\begin{array}{c}63,8 \pm \\
9,6\end{array}$ & $\begin{array}{c}61,8 \pm \\
13,6\end{array}$ & \\
\hline $\begin{array}{l}\text { Hemoglobina } \\
(\mathrm{g} / \mathrm{dl})^{*}\end{array}$ & $\begin{array}{c}8,2 \pm \\
1,7\end{array}$ & $\begin{array}{c}8,6 \pm \\
2,1\end{array}$ & 0,636 & $\begin{array}{c}8,5 \pm \\
2\end{array}$ & $\begin{array}{c}8,9 \pm \\
2,4\end{array}$ & 0,68 & $\begin{array}{c}7,3 \pm \\
4,1\end{array}$ & $\begin{array}{c}7,9 \pm \\
7,6\end{array}$ & 0,605 \\
\hline $\begin{array}{l}\text { Creatinina } \\
(\mathrm{mg} / \mathrm{dl})^{*}\end{array}$ & $\begin{array}{c}2,6 \pm \\
2,1\end{array}$ & $\begin{array}{c}2,1 \pm \\
1,8\end{array}$ & 0,578 & $\begin{array}{c}2,4 \pm \\
2\end{array}$ & $\begin{array}{c}1,6 \pm \\
0,6\end{array}$ & 0,37 & $\begin{array}{c}2,4 \pm \\
1,7\end{array}$ & $\begin{array}{c}2,1 \pm \\
1,9\end{array}$ & 0,636 \\
\hline $\begin{array}{l}\text { Albumina } \\
(\mathrm{g} / \mathrm{dl})^{*}\end{array}$ & $\begin{array}{c}2,9 \pm \\
0,6\end{array}$ & $\begin{array}{c}3,2 \pm \\
0,7\end{array}$ & 0,319 & $\begin{array}{c}3,2 \pm \\
0,7\end{array}$ & $\begin{array}{c}2,7 \pm \\
0,6\end{array}$ & 0,15 & $\begin{array}{c}3,2 \pm \\
0,6\end{array}$ & $\begin{array}{c}3,2 \pm \\
0,7\end{array}$ & 0,921 \\
\hline $\begin{array}{l}\text { Cálcio lonizável } \\
(\mathrm{mg} / \mathrm{dl})^{*}\end{array}$ & $\begin{array}{c}1,6 \pm \\
0,5\end{array}$ & $\begin{array}{c}1,3 \pm \\
0,2\end{array}$ & 0,033 & $\begin{array}{c}1,4 \pm \\
0,3\end{array}$ & $\begin{array}{c}1,6 \pm \\
0,6\end{array}$ & 0,19 & $\begin{array}{c}1,6 \pm \\
0,4\end{array}$ & $\begin{array}{c}1,3 \pm \\
0,1\end{array}$ & 0,028 \\
\hline $\begin{array}{l}\beta 2- \\
\text { microglobulina } \\
(\mathrm{mg} / \mathrm{dl})^{*}\end{array}$ & $\begin{array}{c}13,4 \pm \\
9,1\end{array}$ & $\begin{array}{c}5,4 \pm \\
2,9\end{array}$ & 0,003 & $\begin{array}{c}6,5 \pm \\
3,9\end{array}$ & $\begin{array}{c}12,5 \pm \\
11,5\end{array}$ & 0,04 & $\begin{array}{c}8,3 \pm \\
1,3\end{array}$ & $\begin{array}{c}8,7 \pm \\
2,4\end{array}$ & 0,605 \\
\hline \multicolumn{10}{|c|}{ Componente Monoclonal } \\
\hline $\operatorname{lgG} n(\%)$ & $2(29)$ & $16(70)$ & & $13(54)$ & $4(66)$ & & $6(50)$ & $12(66)$ & \\
\hline $\operatorname{lgA} n(\%)$ & $1(14)$ & $3(13)$ & & $3(16)$ & $1(17)$ & & $1(16)$ & $2(10)$ & \\
\hline $\begin{array}{l}\text { Cadeia leve } \\
\text { kappa n (\%) }\end{array}$ & $6(86)$ & $15(65)$ & & $18(75)$ & $4(66)$ & & $8(65)$ & 14) (78) & \\
\hline $\begin{array}{l}\text { Cadeia leve } \\
\text { lambda n (\%) }\end{array}$ & $1(14)$ & $8(35)$ & & $6(25)$ & $2(34)$ & & $4(25)$ & $4(22)$ & \\
\hline \multicolumn{10}{|c|}{ Estadiamento de Durie\&Salmon } \\
\hline $\ln (\%)$ & 0 & $1(4)$ & & $1(4)$ & 0 & & $1(8,0)$ & $1(5)$ & \\
\hline II n (\%) & 0 & $1(4)$ & & $1(4)$ & $1(18)$ & & $1(8)$ & $1(5)$ & \\
\hline IIIA n (\%) & $3(42)$ & $14(62)$ & & $13(54)$ & $3(50)$ & & $7(59)$ & $10(56)$ & \\
\hline IIIB n (\%) & $4(58)$ & $7(30)$ & & $9(36)$ & $2(32)$ & & $3(25)$ & $6(34)$ & \\
\hline \multicolumn{10}{|l|}{ ISS } \\
\hline I n (\%) & & $5(25)$ & & 0 & $1(10)$ & & $1(8,0)$ & $1(5,0)$ & \\
\hline II n (\%) & 1 (14) & $7(30)$ & & $4(16)$ & $2(30)$ & & $3(25)$ & $2(26)$ & \\
\hline III n (\%) & $6(86)$ & $11(47)$ & & $20(84)$ & $3(50)$ & & $8(67)$ & $12(68)$ & \\
\hline
\end{tabular}

*Valores expressos por média e desvio padrão. ISS: Sistema Internacional de Estadiamento.

Nos pacientes que expressavam mais de dois fenótipos aberrantes mieloides, foi observado que os mesmos apresentavam níveis mais elevados de creatinina sérica que o grupo que não expressava os antígenos mieloides $(2,7 \mathrm{mg} / \mathrm{dl}$ e $1,5 \mathrm{mg} / \mathrm{dl}$, respectivamente) $(p=0,04)$. Os demais antígenos (CD10, CD19, CD20, CD22, CD45 e HLA-DR) não mostravam nenhuma associação.

\section{Discussão}

Os plasmócitos mielomatosos, assim como os normais, são caracterizados por expressar os antígenos CD138 ${ }^{+++}$ e $\mathrm{CD} 38^{++}$, porém os plasmócitos de indivíduos normais caracterizam-se pela presença dos antígenos de superfície associados à linhagem B, como $\mathrm{CD} 19^{++}, \mathrm{CD} 20^{++}$e CD22 ${ }^{++}$, 
e não expressam os antígenos CD13, CD33, CD28, CD56 e CD117. Neste aspecto, os plasmócitos do mieloma múltiplo diferem tanto dos normais quanto das demais doenças linfoproliferativas por apresentarem baixa frequência dos marcadores associados às células $B(C D 10, C D 19, C 20$, CD22) e por expressarem moléculas de outras linhagens denominadas de fenótipos aberrantes ${ }^{(5)}$.

Desde a década de 1990 são relatados fenótipos aberrantes nos plasmócitos mielomatosos, sendo os fenótipos $\mathrm{CD} 56^{+++}, \mathrm{CD} 117^{++}, \mathrm{CD} 33^{++}, \mathrm{CD} 13^{++}$e CD28 ${ }^{++}$os mais comumente encontrados. A maioria dos casos de MM exibe forte expressão do CD56 e fraca expressão dos antígenos CD45. Alguns estudos demonstram que cerca de $75 \%$ dos casos expressam o $\mathrm{CD}_{56} 6^{++}$e que apenas $35 \%$ exibem o CD11 $7^{++}$. Poucos estudos têm reportado a expressão do CD33 em plasmócitos clonais, sendo este marcador aberrante associado às células mieloides expresso em apenas $12 \%$ dos casos de MM. Já o antígeno associado à ativação de linfócitos T CD28 é expresso em aproximadamente 41\% dos pacientes portadores de $\mathrm{MM}^{(10,11,15)}$.

Em nosso estudo foi observada alta frequência dos fenótipos aberrantes nos plasmócitos mielomatosos, sendo que $60 \%$ dos casos expressaram o CD117 com moderada a forte intensidade e que o antígeno CD28 foi encontrado em apenas $20 \%$ dos casos com alta expressão antigênica. Em relação à frequência de expressão do antígeno de superfície CD33 foi relatado que cerca de $30 \%$ dos pacientes estudados exibiram forte positividade para este marcador.

O antígeno CD56 (molécula de adesão de célula neural [NCAM]) está envolvido na embriogênese normal dos axônios. Comumente este marcador está presente em células natural killer e NCAM. Contudo, vem-se observando alta frequência deste marcador em plasmócitos mielomatosos e grande associação entre a presença de $\operatorname{CD} 56^{+++}$e a agressividade da doença. Além disso, foi detectado que os pacientes portadores de MM com o fenótipo CD56 apresentavam doença avançada, sendo este achado fenotípico correlacionado com pior prognóstico em pacientes com MM tratados com quimioterapia convencional $(13,14,17,19)$. Entretanto, estudos de Hundermer et al. ${ }^{(7)}$ relatam que a negatividade do CD56 não pode ser considerada um marcador relacionado com mau prognóstico, pois, quando foi comparada a sobrevida livre de doença dos pacientes mielomatosos $\mathrm{CD} 56^{+++}$com a dos pacientes $\mathrm{CD} 56$ - submetidos a altas doses de quimioterapia, observou-se que os dois grupos possuíam sobrevidas similares.

Nossos achados mostram que os plasmócitos do MM expressam frequentemente o marcador CD56 $6^{++}$. Já os pacientes que perdem o antígeno CD56 exibem níveis séricos mais altos de $\beta 2 \mathrm{M}$ e cálcio ionizável que os pacientes com CD56 ${ }^{+++}$, confirmando que a ausência desta molécula de adesão está relacionada com estágios mais agressivos da doença. Além disso, o presente estudo também confirmou que a combinação entre o $\mathrm{CD} 56^{+++}$e o CD19- permite melhor identificação dos plasmócitos mielomatosos, visto que o CD56 está presente na maioria dos casos de MM e que o CD19 está ausente em mais de 95\% dos pacientes estudados.

O antígeno CD28 é uma proteína de superfície expressa em linfócitos $T$, sendo um importante antígeno para a ativação destas células. Este marcador não é expresso em plasmócitos normais, porém está presente em de $41 \%$ dos casos de MM. A expressão aberrante deste antígeno tem sido encontrada com maior reatividade nas fases mais avançadas da doença e está associada a casos de maior agressividade e progressão(18).

A frequência do fenótipo $\mathrm{CD}_{28} 8^{++}$(20\%) em nosso estudo foi mais baixa do que os dados da literatura. Contudo, mesmo com a baixa frequência deste antígeno, foi possível associar os casos $C D 28^{++}$a altos níveis de $\beta 2 \mathrm{M}$, sugerindo que esta expressão aberrante deve estar relacionada com estágios mais avançados da doença e com maior massa tumoral.

\section{Conclusão}

A partir desta pesquisa pode-se concluir que os plasmócitos mielomatosos podem ser caracterizados por um perfil imunofenotípico por meio da expressão e ausência de alguns marcadores (CD138 $8^{+++}, \mathrm{CD}^{++}, \mathrm{CD}^{+} 5^{+}$ou CD45; CD56 $\left.{ }^{+++}, \mathrm{CD} 19-\mathrm{CD}^{-} 17^{++}\right)$. Os fenótipos CD56- e CD28 ${ }^{++}$ estão associados a estágios avançados do mieloma múltiplo, sendo a imunofenotipagem por citometria de fluxo multiparamétrica uma ferramenta extremamente útil na diferenciação entre as células plasmáticas reacionais e os plasmócitos clonais. 


\section{Referências}

1. ALMEIDA, J. et al. High-sensitive immunophenotyping and DNA ploidy studies for the investigation of minimal residual disease in multiple myeloma. Br J Haematol, v. 107, n. 1, p. 121-31, 1999.

2. BAITALLE, R.; HAROUSSEAU, J. L. Multiple Myeloma. N Eng J Med, v. 336, n. 23, p. 1657-64, 1997.

3. DURIE, B. G.; SALMON S. E. A clinical staging system for multiple myeloma. Correlation of measured myeloma cell mass with presenting clinical features, response to treatment, and survival. Cancer, v. 36, n. 3, p. 842-54, 1975.

4. GREIPP, P. R. et al. International staging system for multiple myeloma. J Clin Oncol, v. 23, n. 15, p. 3412-20, 2005.

5. GUIKEMA, J. E. et al. Hetetogeneity in the multiple myeloma tumor clone. Leuk Lymphoma, v. 45, n. 5, p. 857-71, 2004.

6. HARADA, H. et al. Phenotypic difference of normal plasma cell from mature myeloma cells. Blood, v. 81, n. 10, p. 2658-63, 1993.

7. HUNDEMER M. et al. Lack of CD56 expression on myeloma cells is not a marker for poor prognosis in patients treated by high dose chemotherapy and is associated with translocation $\mathrm{t}(11 ; 14)$. Bone Marrow Transplant, v. 40, n. 11, p. 1033-37, 2007.

8. KYLE, R. A.; RAJKUMAR, S. V. Criteria for diagnosis, staging, risk stratification and response assessment of multiple myeloma. Leukemia, v. 23, n. 1, p. 3-9, 2009.

9. LIMA, M. et al. Immunophenotypic aberrations, DNA content and cell cycle analysis of plasma cells in patientes with myeloma and monoclonal gammopathies. Blood Cells, Molecules and Diseases, v. 26, n. 6, p. 634-45, 2000.

10. LIN, P. et al. Flow Cytometric immunophenotypic analysis of 306 cases of multiple myeloma. Am J Clin Pathol, v. 121, n. 4, p. 482-88, 2004.

11. MATEO, G. et al. Genetic abnormalities and patterns of antigenic expression in multiple myeloma. Clin Cancer Res, v. 11, n. 10, p. 3361-7, 2005.
12. OCQUETEAU, M. etal. Immunophenotypic characterization of plasma cells from monoclonal gammopathy of undetermined significance patients. Implications for the differential diagnosis between MGUS and multiple myeloma. Am J Clin Pathol, v. 152, n. 6, p. 1655-65, 1998.

13. PELLAT-DECEUNYNCK, C. et al. Adhesion molecules on human myeloma cells: significant chances in expression related to malignation tumor spreading and immortalization. Cancer Research, v. 55, n. 16, p. 3647-53, 1995.

14. PELLAT-DECEUNYNCK, C. et al. The absence of CD56 (NCAM) on malignant plasma cells is hallmark of plasma cell leukemia and of a special subset of multiple myeloma. Leukemia, v. 12, n. 12, p. 1977-82, 1998.

15. RAJA, K. R. M.; KOVAROVA L.; HAJEK R. Review of phenotypic markers used in flow cytometric analysis of MGUS and MM, and applicability of flow cytometry in other plasma cell disorders. Br J Haematol, v. 149, n. 3, p. 334-51, 2010.

16. RAWSTRON, A. C. et al. Report of the European Myeloma Network on multiparametric flow cytometry in multiple myeloma and related disorders. Haematologica, v. 93, n. 3, p. 431-38, 2008.

17. SAHARA, N.; TAKESHITA, A. Prognostic significance of surface markers expressed in multiple myeloma: CD56 and other antigens. Leuk Lymphoma, v. 45, n. 1, p. 61-5, 2004.

18. SHAPIRO, S. M.; MOLLLENAUER, M. N.; WEISS A. Endogenous CD28 expressed on myeloma cells upregulates interleukin-8 production: implications for multiple myeloma progression. Blood, v. 98, n. 1, p. 187-93. 2001.

19. VAN CAMP, B. et al. Plasma cells in multiple myeloma express a natural killer cell-associated antigen: CD56 (NKH-1, Leu-19). Blood, v. 76, n. 2, p. 377-82, 1990 